\title{
Does history-taking help predict rabies diagnosis in dogs?
}

\author{
Veera Tepsumethanon, Boonlert Lumlertdacha, Channarong Mitmoonpitak \\ Veterinary Diagnostic Laboratory, Queen Saovabha Memorial Institute, The Thai Red Cross Society, \\ Bangkok 10330, Thailand
}

\begin{abstract}
Background: Rabies is a fatal disease. However, dogs are the principal vehicle for rabies transmission of human. A little information about pre-morbid behavior in rabid dogs could be found in the literature.

Objective: Assess the value of history taking in predicting rabies diagnosis in dogs, and identify the percentage of rabies positives by history taking in a prospective study.

Materials and methods: Studies were conducted at the Rabies Diagnostic Unit, Queen Saovabha Memorial Institute, Thai Red Cross Society between 2002 and 2008. Historical data were collected prospectively from 153 live rabies suspected dogs on admission to the diagnostic facility.

Results: Rabies was found in $14 \%$ to $80 \%$ of dogs with completed questionnaires except for dogs less than one month old, not sick or sick for more than 10 days.

Conclusion: History taking does not help in decision-making for rabies post-exposure prophylaxis of humans.
\end{abstract}

Keywords: Clinical diagnosis, history-taking, observation, rabid dog

The Queen Saovabha Memorial Institute of the Thai Red Cross Society has served as the principal animal rabies diagnostic center for the Bangkok Metropolitan region and neighboring provinces since 1950. Activities are laboratory diagnosis using two techniques, fluorescent antibody test (FAT) [1] and mouse inoculation test (MIT) [2] that include intravitam diagnosis by clinical examination with 10 day observation and providing pre and post-exposure prophylaxis for animals. Results of these activities are immediately available for the physicians at the postexposure animal bite center, which is part of the institute. Annually, 400 dead and 30 live rabies suspected animals are examined. Most rabies suspected animals were dogs (70\%), which represent $95 \%$ of laboratory proven rabid animals. In Thailand, dogs are the main vehicle of the disease and cause of post-exposure prophylaxis and of human rabies deaths.

Correspondence to: Veera Tepsumethanon, Veterinary Diagnostic Laboratory, Queen Saovabha Memorial Institute, The Thai Red Cross Society, Bangkok 10330, Thailand. E-mail: tepsumethanonv@yahoo.com
This is virtually the same situation as in other canine rabies endemic countries of Asia and Africa [3-6].

We could find little information in the literature concerning data about pre-morbid behavior in rabid dogs that proved helpful for diagnosis and patient management decision making. Therefore, the authors collected and analyzed prospectively histories taken from owners on the day of admission of live rabies suspected dogs.

\section{Materials and methods}

One hundred fifty three live dogs had either bitten humans and/or animals or had shown altered behavior or abnormal physical signs and had been submitted for veterinary observation between 2002 and 2008 . After clinical examination and recording of case histories, the dogs were kept in individual cages and observed daily for 10 days. If the dog died during the observation period, brain necropsy was performed using the standard fluorescent antibody (FAT) and mouse inoculation tests (MIT). MIT was only performed if the FAT was negative $[1,2]$. If dogs survived $\geq 10$ days of observation, they were considered 
not rabid based on previously published data [7-9] and were released to their owners or transferred to a municipal dog shelter.

Figure 1 and 2 show the two clinical presentations of rabies and the removal of brain for laboratory examination, respectively.

\section{Results}

Seventy-four dogs were found to be rabid by necropsy and FAT. Rabid dogs owner answered almost all the history-taking questions (14-80\%) except for three groups of dogs less than one month old, not sick or sick for more than 10 days (Table 1, Fig. 3).

Some rabid suspected dogs were found positive on necropsy (46-64\%). Those dogs were either owned or stray, may have bitten a human or an animal during the past 10 days, and may have been standing or lying down on admission. However, they were all older than one or more month old, did not show the "Circling" sign within one week prior to admission, were never immunized with rabies vaccine, caused unprovoked bites, and were bitten by stray dogs during the past two months. Furthermore, all of the dogs were sick or showing abnormal behavior on the day of submission, have been sick for less than 10 days, and have had a gradual onset. A high percentage of rabies was found in dogs with progressive sickness with rapidly downward course and dogs in an area where a rabid dog was found during the past two months (69\% and $80 \%)$.
A

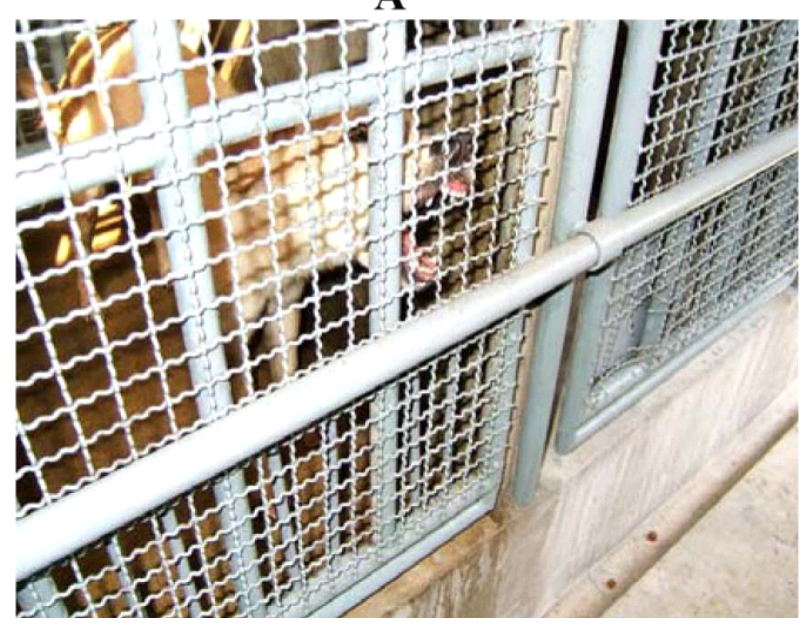

B

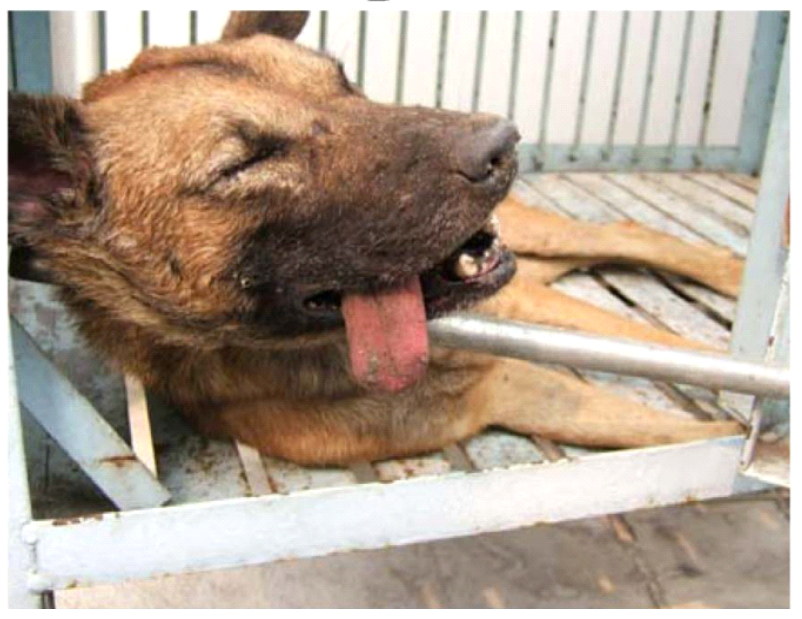

Fig. 1 A: Furious rabies. Biting cage. B: Dumb rabies. Drooping jaw.

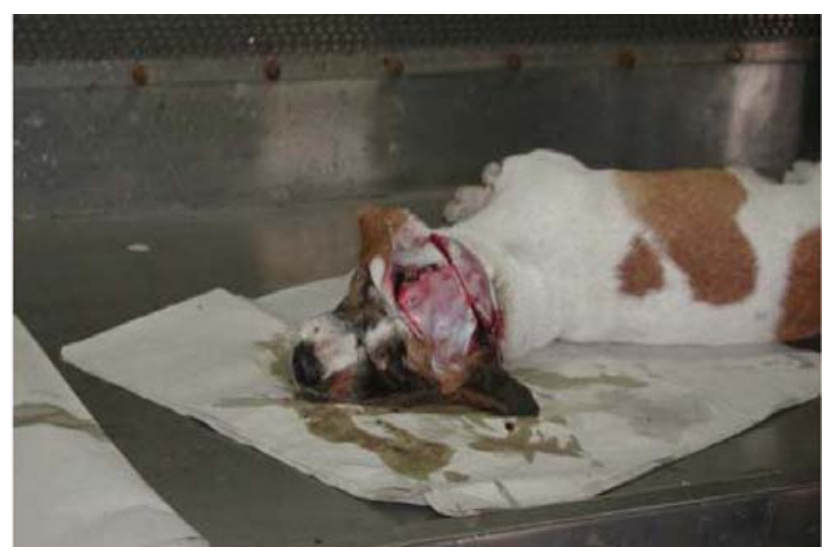

Fig. 2 Removal of brain for FAT. 
Table 1. Historical data from 153 live rabies suspected dogs.

Question and answer

\section{Routine diagnosis by FAT or having} survived $>10$ days

\begin{tabular}{lll}
\hline $\begin{array}{l}\text { Positive } \\
(n=74)\end{array}$ & $\begin{array}{l}\text { Negative } \\
(n=79)\end{array}$ & $\begin{array}{l}\% \text { of } \\
\text { positive }\end{array}$ \\
\hline
\end{tabular}

1. Status?

2. Previously rabies vaccinated?

3. Bite during the past 10 days?

4. Provoked or unprovoked bite?

5. Bitten by stray dogs during the past 2 months?

6. A rabid dog found in the same area during the past 2 months?

7. Dog's condition on admission?

8. Dog's age?

9. Sick or abnormal behavior?

10. Duration of sickness or abnormal behavior?

11. Onset?

12. Progressive sickness (without treatment) during the past 3-5 days?

13. ***"Circling" sign within 1 week prior to admission?
Owned

Stray

Unknown

*Yes

No

Unknown

Bitten

Not bitten

Unknown

Provoked

Unprovoked

Yes

No

Unknown

Yes

No

Unknown

Standing

Lying down

$<1$ month

$\geq 1$ month

Unknown

Not sick or Normal

behavior

Sick or Abnormal $\quad 73$

behavior

Unknown

$>10$ days

$<10$ days

Unknown

**Acute

Gradual

Unknown

Stable or improve

Downward

Unknown

Yes

No

Unknown

1

$46 \quad 51$

47

26

46

16

31

2

33

36

31

62

49

48

50

38

18

64

58

32

52

80

40

53

46

54

0

49

33

0

23

59

51

17

0

63

17

29

62

23

25

69

33

14

52

47

$*$ last vaccination: $<6$ months, Positive $=8$, Negative $=12, \%=40 ;>6$ months, Positive $=8$, Negative $=24$, $\%=25$. $* *$ Acute means having no prodrome. $* * *$ Circling sign means one-way walking in circles and hitting against the cage "as if blind". 


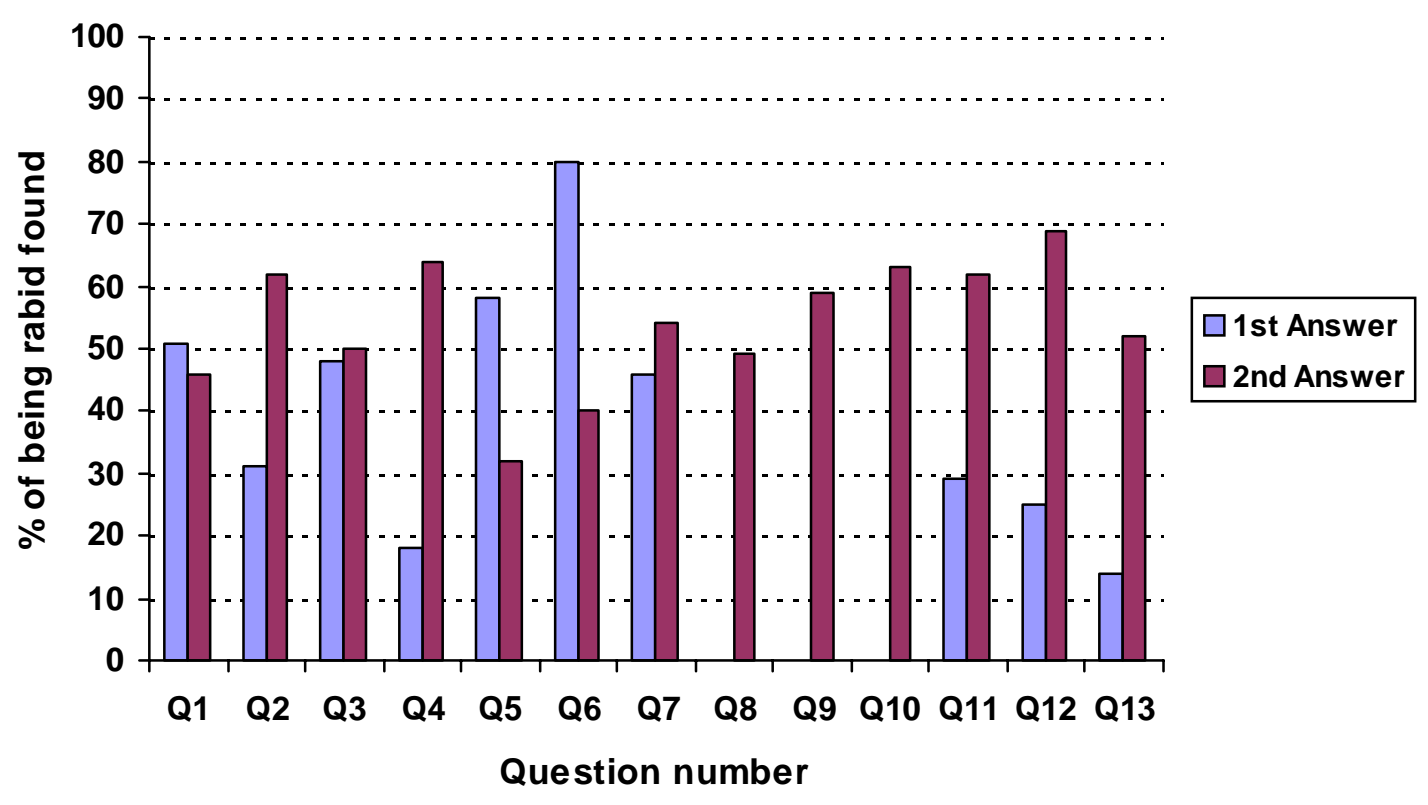

Fig. 3 The percentages of being rabid found in each answer of the 13 questions.

\section{Discussion}

Live dogs that show nervous signs, or have bitten humans and/or animals or have altered behavior will have a $14-80 \%$ chance of being rabid in a canine rabies endemic region (Table 1). No rabid dogs were found that were less than one month old, not sick or sick for more than 10 days. This supports prior studies [7-9] and is clinically useful data for diagnosis in suspect dogs. Provoked or unprovoked bites represent approximately the same chance of infection. This confirms prior studies of dog bites [10,11].

In the groups with low probability of rabies, acute onset of sickness (29\%), sickness during the past three to five days but remained stable or improving $(25 \%)$, or showing the "Circling" sign within one week (14\%) indicated a low risk or rabies. However, these results are not reliable $[8,9]$. There is the probability that some of the owners gave incorrect answers. Rabies does not usually develop clinical signs without a prodromal phase or with acute onset. Rabies symptoms and signs are not stable unless the dog receives supportive treatment such as fluid therapy and sedation. Rabies will not improve by the use of antibiotics. "Circling" is a specific sign of canine distemper [8,9].

Thirty one percent of dogs that had been previously immunized with rabies vaccine found rabid (Table 1). This number appears high and might be influenced by the quality of vaccine and incorrect information. The authors divided these immunized dogs into two groups by timing of the last rabies vaccination before death, $<6$ and $>6$ months ago among which $40 \%$ and $25 \%$ were found rabid by FAT, respectively (Table 1). We suspect that in the former group vaccination may have occurred during the incubation period (too late to be effective). Owners might not know that such vaccination was not timely enough and not adequate for post-exposure prophylaxis. Some owners may also have given incorrect answers since they did not bring the vaccine certification with the dogs. In the United States, rabies is uncommon in vaccinated dogs and cats but can occur since $4.9 \%$ rabid dogs and $2.6 \%$ rabid cats had a history of rabies vaccination [12]. In addition, the immune response to rabies vaccine in unvaccinated dogs will be affected by the route of inoculation (intramuscular or subcutaneous) and the number of shots in the first year of vaccination [13-16].

None of the authors has any conflicts of interest to report.

\section{References}

1. Dean DJ, Abelseth MK, Atanasiu P. The fluorescent antibody test. In: Meslin FX, Kaplan MM, Koprowski $\mathrm{H}$, eds. Laboratory Techniques in Rabies. $4^{\text {th }}$ ed. Geneva:World Health Organization, 1996. p. 88-95.

2. Koprowski H. The mouse inoculation test. In: Meslin 
FX, Kaplan MM, Koprowski H, eds. Laboratory Techniques in Rabies. $4^{\text {th }}$ ed. Geneva:World Health Organization, 1996. p. 80-87.

3. Anonymous. Rabies statistics in animals. Annual Epidemiology Surveillance Report, Bureau of Disease Control and Veterinary Services, Department of Livestock Development, Ministry of Agriculture and Cooperatives, Thailand, 2005-2007.

4. World Health Organization. WHO Expert Consultation on Rabies. First report. WHO Technical Report Series 931. Geneva:World Health Organization, 2005.

5. Wilde H, Hemachudha T, Khawplod P, Tepsumethanon V, Wacharapluesadee S, Lumlertdacha B. Rabies 2007: perspective from Asia. Asian Biomed. 2007; 1: 345-57.

6. Garden D. Rabies research in Thailand. Asian Biomed. 2009; 3:213-20.

7. Tepsumethanon V, Lumlertdacha B, Mitmoonpitak C, Sitprija V, Meslin F-X, Wilde H. Survival of naturally infected rabid dogs and cats. CID. 2004; 39:278-80.

8. Tepsumethanon V, Wilde H, Meslin F-X. Six criteria for rabies diagnosis in living dogs. J Med Assoc Thai. 2005; 88:419-22.

9. Tepsumethanon V, Wilde H, Sitprija V. Ten-day observation of live rabies suspected dogs. In: Dodet B, Fooks AR, Muller T, et al, editors. Towards the elimination of rabies in Eurasia. Dev Biol. Basel: Karger, 2008, Vol 131, p. 543-6.

10. Siwasantiwat D, Lumlertdacha B, Polsuwan C, Hemachuda T, Chutivongse S, Wilde H. Rabies: is provocation of the biting dog relevant for risk assessment? Transact Roy Soc Trop Med Hyg. 1992; 86:443.

11. Dutta JK. Bite by a dog under provocation: is it free from risk? J Indian Med Assoc. 2002; 100:330-1.

12. Murray KO, Holmes KC, Hanlon CA. Rabies in vaccinated dogs and cats in the United States, $1997-$ 2001. J Am Vet Med Assoc. 2009; 235:691-5.

13. Tepsumethanon W, Polsuwan C, Lumlertdacha B, Khawplod P, Hemachudha T, Chutiwongse S, Wilde $\mathrm{H}$, Chiewbamrungkiat M, Phanuphak P. Immune response to rabies vaccine in Thai dogs, a preliminary report. Vaccine. 1991; 9:627-30.

14. Sage G, Khawplod P, Wilde H, Lobaugh C, Hemachudha T, Tepsumethanon W, et al. Immune response to rabies vaccine in Alaskan dogs: failure to achieve a consistently protectively antibody response. Transact Roy Soc Trop Med Hyg. 1993;87:593-5.

15. Cliquet F, Verdier Y, Sagné L, Aubert M, Schereffer JL, Selve $M$, et al. Neutralising antibody titration in 25,000 sera of dogs and cats vaccinated against rabies in France, in the framework of the now regulations that offer an alternative to quarantine. Rev Sci Tech. 2003, 22:857-66.

16. Mansfield KL, Burr PD, Snodgrass DR, Sayers R, Fooks AR. Factors affecting the serological response of dogs and cats to rabies vaccination. Vet Rec. 2004; 154:423-6. 